\title{
Two-Dimensional Treatment of Mode Theory of the Propagation of VLF Radio Waves
}

\author{
James R. Wait
}

\section{Contribution From the Central Radio Propagation Laboratory, National Bureau of Standards, Boulder, Colo.}

\begin{abstract}
This paper is partly of a tutorial nature. The intended purpose is to exploit the essential two-dimensional nature of wave propagation in the earth-ionosphere waveguide. It is shown that, without resorting to erudite arguments in the complex plane, the usual working formulas of VLF mode theory may be derived directly from orthogonality considerations. Furthermore, the physical insight gained by the present development immediately sug gests how the formulas may be generalized to an earth-ionosphere waveguide of nonuniform width.
\end{abstract}

\section{Introduction}

In the theory of terrestrial radio propagation, the earth is represented by a spherical body and the atmosphere is usually idealized by concentric spherical layers. In this sense the problem is formulated as a three-dimensional one. However, in subsequent developments and in the reduction to useful formulas, approximations are usually made which display the inherent twodimensional character of the problem. Typical of these approximations are the replacement of the spherical wave functions by Airy functions and the Legendre functions by leading terms in their asymptotic expansions.

It is the purpose of the present paper to develop the final formulas directly from a twodimensional formulation. This should help dispel any doubts concerning the validity of the approximated three-dimensional solutions. A demonstration such as this is timely since a twodimensional laboratory model has been used recently to study VLF propagation in a spherical waveguide. ${ }^{1}$ At the same time we shall discuss the excitation factors, height-gain functions [Spies and Wait, 1963], and mode conversion coefficients [Wait, 1962a] for the two-dimensional model. For the purposes of this paper, the ionosphere is assumed to be equivalent to an isotropic medium.

\section{Primary Fields}

In formulating the radio problem in spherical coordinates, it is customary to choose the source as a radial electric dipole or a radial magnetic dipole. By an appropriate superposition, the fields of an arbitrary source may be determined. In a two-dimensional model it is more convenient to work with line sources. For example, in place of a radial electric dipole on a spherical surface it is convenient to employ a line dipole source. To introduce the subject the fields, of such a line dipole located in free space, are derived from basic principles.

The line source consists of a uniform distribution of vertical electric dipoles. The situation is illustrated in figure 1 where the source current density is $I$ amps per meter, for a strip of width $d l$, along the $x$ axis. Because the source current has only a $z$ component, the resulting Hertz vector also has only a $z$ component, $\Pi_{z}$. Clearly, the Hertz vector of the distribution is obtained by integrating the contributions from the distribution of vertical electric dipoles; thus [Wait, 1959]

$$
\Pi_{z}=\int_{-\infty}^{+\infty} \frac{I d l}{4 \pi i \epsilon_{0} \omega} \frac{e^{-i k r}}{r} d x^{\prime},
$$

\footnotetext{
1 Microwave model experiments being conducted by the author's colleagues E. Bahar and S. Maley in the Department of Electrical Engineering at the University of Colorado.
} 


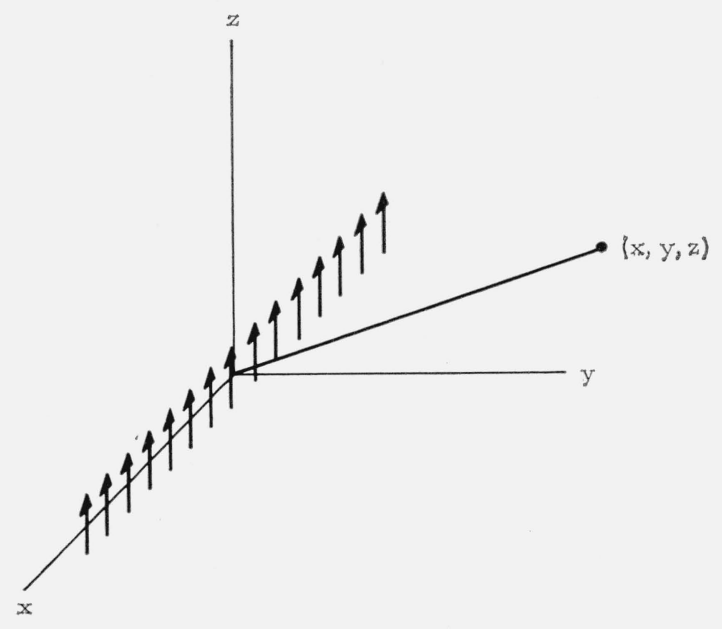

FiguRe 1. Line dipole source and coordinate system employed for the discussion of primary fields.

where $k=2 \pi /$ wavelength,

$r=\left[\left(x-x^{\prime}\right)^{2}+y^{2}+z^{2}\right]^{1 / 2}$,

and $\epsilon_{0}=8.854 \times 10^{-12}$. The integration with respect to $x^{\prime}$ may be readily carried out, to give [Wait, 1959]

$$
\Pi_{z}=\frac{I d l}{2 \pi i \epsilon_{0} \omega} K_{0}(i k \rho)
$$

where $\rho=\left(y^{2}+z^{2}\right)^{1 / 2}$ and $K_{0}$ is a modified Bessel function of order zero. The resulting magnetic field, which has only an $x$ component, is obtained from

$$
H_{x}=\frac{i k^{2}}{\mu_{0} \omega} \frac{\partial \Pi_{z}}{\partial y}
$$

where $\mu_{0}=4 \pi \times 10^{-7}$. In the far zone, where $k \rho>>1$, this reduces to

$$
H_{x} \cong-\frac{I d l(i k)^{1 / 2}}{2(2 \pi \rho)^{1 / 2}} e^{-i k_{\rho}} \sin \alpha,
$$

where $\sin \alpha=y / \rho$. It is of interest to observe that the radiation pattern characterized by $\sin \alpha$, is identical to the pattern of an isolated vertical dipole. However, in the case of a line dipole source, the radiation field is a cylindrical wave whose amplitude varies inversely as the square root of distance. This is to be contrasted with the radiation field of a point electric dipole which is a spherical wave with an inverse distance variation of the amplitude. An obvious point of similarity between the line dipole and the point dipole is the common phase factor $\exp (-i k \rho)$, occurring in the radiation fields.

The preceding rather elementary derivation is based on a prior knowledge of the expression for the Hertz vector for a point dipole. It is instructive to obtain the same final formula for $H_{x}$ by using a transform method.

The starting point is to recognize that $H_{x}$ must satisfy the wave equation

$$
\left(\frac{\partial^{2}}{\partial y^{2}}+\frac{\partial^{2}}{\partial z^{2}}+k^{2}\right) H_{x}=0
$$

Solutions are of the form $\exp ( \pm i \lambda z) \exp ( \pm u y)$ where $u=\left(\lambda^{2}-k^{2}\right)^{1 / 2}$ and $\lambda$ is a parameter. A general solution is constructed by writing

$$
H_{x}=\int_{-\infty}^{+\infty} F(\lambda) e^{-i \lambda z} e^{\mp u y} d \lambda
$$


where $F(\lambda)$ is an unknown function and where the contour of integration is along the whole real axis of $\lambda$. The radical is to be chosen in $u$ such that $\operatorname{Re} u>0$. Thus, the minus sign in the exponent is to be employed when $y>0$ and the positive sign is chosen when $y<0$. Difficulties with branch points on the contour are avoided by allowing $k$ to have a very small but finite negative imaginary part (i.e., - Imag. $k>0$ ).

The function $F(\lambda)$ is obtained by noting that $H_{x}$ must become numerically equal to $-I / 2$ at $z=0$ and as $y$ tends to zero from positive values. Thus

$$
\left.H_{x}\right]_{y=0^{+}} \simeq-\frac{I}{2} \delta(z) d l,
$$

where $\delta(z)$ is the unit impulse function at $z=0$. It should also be noted that

$$
\left.H_{x}\right]_{y=0^{-}} \simeq+\frac{I}{2} \delta(z) d l .
$$

Employing the spectral representation of the unit impulse function, i.e.,

$$
\delta(z)=\frac{1}{2 \pi} \int_{-\infty}^{+\infty} e^{-i \lambda z} d \lambda,
$$

it follows from (6) and (7a) that

$$
F(\lambda)=-\frac{I d l}{4 \pi}
$$

Thus

$$
H_{x}=-\frac{I d l}{4 \pi} \int_{-\infty}^{+\infty} e^{-i \lambda z} e^{-u y} d \lambda
$$

for $y>0$. This integral in (10) may be evaluated by noting that

$$
K_{0}(i k \rho)=\frac{1}{2} \int_{-\infty}^{+\infty} e^{-i \lambda z} e^{-u y} u^{-1} d \lambda
$$

which may be differentiated under the integral sign with respect to $y$. As a result,

$$
H_{x}=-\frac{i k I d l}{2 \pi} K_{1}(i k \rho) \sin \alpha
$$

where $\sin \alpha=y / \rho$ and $K_{1}$ is the modified Bessel function of order one. In the far zone, where $k \rho>>1$, it follows that

$$
H_{x} \cong-\frac{(i k)^{1 / 2} I d l}{2(2 \pi \rho)^{1 / 2}} e^{-i k \rho} \sin \alpha
$$

which is identical to (4) as it should be.

\section{Fields in the Concentric Cylindrical Waveguide}

To come to grips with the general problem, a rather simple model is chosen. The waveguide region consists of two concentric cylindrical surfaces of radii $a$ and $a+h$, respectively, as indicated in figure $2 \mathrm{a}$. In terms of a cylindrical coordinate system $(r, \theta, z)$, these bounding surfaces are defined by $r=a$ and $r=a+h$ where the tangential fields are assumed to satisfy impedance-type boundary conditions. The field variations in the $z$ direction are taken to be zero so that the modes in the structure may be either TM (transverse magnetic) or TE (transverse electric). Only the TM modes will be considered here since their analog in VLF radio propagation is of greatest practical interest. The analysis for the TE modes is almost identical.

For the TM modes in such a cylindrical region, the magnetic field has only an axial or $z$ component which is denoted $H$ (without writing the subscript $z$ ). From Maxwell's equations, 


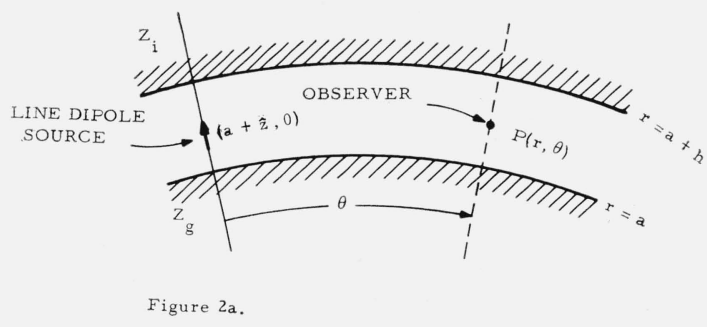

Figure 2a. Concentric cylindrical model and conventional coordinate system $(\mathrm{r}, \theta)$.

it is seen that the electric field has only $r$ and $\theta$ components.

The magnetic field in some aperture plane $\theta=\theta_{0}, a+h>r>a$ is now assumed to be known and designated by $H\left(\theta_{0}, r\right)$, being independent of $z$. The boundary conditions for $H(\theta, r)$ are

$$
\begin{aligned}
& E_{\theta}=-Z_{g} H \text { at } r=a, \\
& E_{\theta}=Z_{i} H \text { at } r=a+h,
\end{aligned}
$$

where $Z_{g}$ and $Z_{i}$ are the respective surface impedances. Within the region $a+h>r>a$, the magnetic field must satisfy the wave equation

$$
\left(\frac{1}{r} \frac{\partial}{\partial r} r \frac{\partial}{\partial r}+\frac{1}{r^{2}} \frac{\partial^{2}}{\partial \theta^{2}}+k^{2}\right) H(\theta, r)=0 .
$$

Solutions are any linear combinations of the form

$$
\begin{aligned}
& H_{\nu}^{(1)}(k r) \\
& H_{\nu}^{(2)}(k r)
\end{aligned} e^{ \pm i \nu \theta}
$$

where $\nu$ is a complex quantity independent of the coordinates. $H_{\nu}^{(1)}$ and $H_{\nu}^{(2)}$ are Hankel functions, of order $\nu$, of the first and second kind, respectively.

Solutions which are periodic require that $\nu$ is an integer. However, this is an unnecessary restriction since individual modes need not be periodic. Instead, the value of $\nu$ will be determined from the boundary conditions. Furthermore, without loss of generality, it is specified that $\operatorname{Re} \nu>0$, and attention is restricted to solutions which behave as $e^{-i \nu \theta}$. This will correspond to waves which are attenuated in the positive $\theta$ direction. The waves propagating in the negative $\theta$ direction are identical in form.

If attention is restricted to large radii of curvature such that both $k a>>1$ and $h / a<<1$ are satisfied, the Hankel functions may be approximated by their Airy function representation. There is a vast amount of literature on this particular subject and here only the final form is quoted. Thus [Wait, 1962b]

where

$$
H_{\nu}^{(2)}(k r) \cong \frac{i}{\pi^{1 / 2}}\left(\frac{2}{k a}\right)^{1 / 3} w_{1}(t-y),
$$

$$
\begin{aligned}
& t=\left(\frac{2}{k a}\right)^{1 / 3}(\nu-k a), \\
& y=\left(\frac{2}{k a}\right)^{1 / 3} k(r-a),
\end{aligned}
$$

and $w_{1}$ is an Airy function. Similarly,

$$
H_{\nu}^{(1)}(k r) \cong-\frac{i}{\pi^{1 / 2}}\left(\frac{2}{k a}\right)^{1 / 3} w_{2}(t-y),
$$




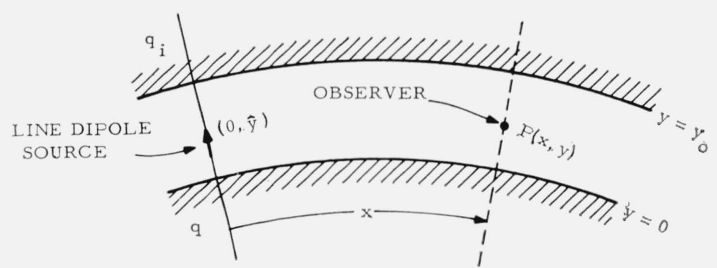

Figure 2b. Concentric cylindrical model with the "natural" coordinates (x, y).

in terms of the Airy function $w_{2}$. In terms of more conventional notation

and

$$
w_{1}(t)=\pi^{1 / 2}[B i(t)-i A i(t)],
$$

$$
w_{2}(t)=\pi^{1 / 2}[B i(t)+i A i(t)]
$$

where the Airy functions $A i$ and $B i$ have been defined and tabulated by Miller [1946]. The functions $w_{1}$ and $w_{2}$ satisfy

$$
\frac{d^{2} w(t)}{d t^{2}}-t w(t)=0
$$

which is known as Stokes' (or Airy's) equation.

To facilitate the subsequent discussion, certain dimensionless parameters are introduced which simplify the notation. These are

$$
\begin{aligned}
x=(k a / 2)^{1 / 3} \theta, x_{0}=(k a / 2)^{1 / 3} \theta_{0}, y=[2 /(k a)]^{1 / 3} k(r-a), y_{0} & =[2 /(k a)]^{1 / 3} k h, q=-i(k a / 2)^{1 / 3} Z_{g} / \eta_{0}, \\
q_{i} & =-i(k a / 2)^{1 / 3} Z_{i} / \eta_{0}, \text { and } \eta_{0}=\left(\mu_{0} / \epsilon_{0}\right)^{1 / 2} \cong 120 \pi .
\end{aligned}
$$

The parameters $x$ and $y$, while describing horizontal and vertical distances, should not be confused with Cartesian coordinates. The quantities $q$ and $q_{i}$ describe conveniently the electrical properties of the bounding walls of the waveguide. The situation is illustrated in figure $2 \mathrm{~b}$.

The (approximate) solutions of (15) are now written as linear combinations of

$$
\begin{aligned}
& w_{1}(t-y) \\
& w_{2}(t-y)
\end{aligned} e^{-i x t},
$$

The discrete values of $t$ (i.e., the eigenvalues), denoted by $t_{n}$, are determined from the boundary conditions. Thus, the resultant field has the form

where

$$
H=H(x, y)=\sum_{n=1,2,3} \ldots b_{n} \Phi\left(t_{n}, y\right) e^{-i\left(x-x_{0}\right) t} n e^{-i k a\left(\theta-\theta_{0}\right)},
$$

$$
\Phi\left(t_{n}, y\right)=w_{1}\left(t_{n}-y\right)+A\left(t_{n}\right) w_{2}\left(t_{n}-y\right),
$$

where $b_{n}$ and $A\left(t_{n}\right)$ are undetermined coefficients. It is evident from (20) that $\Phi$ satisfies

$$
\left(\frac{d^{2}}{d y^{2}}-t+y\right) \Phi(t, y)=0 \text {. }
$$

The boundary conditions given explicitly by (14a) and (14b) may be written in the form

$$
\left.\frac{d}{d y} \Phi(t, y)\right]_{y=0}+q \Phi(t, 0)=0
$$


and

$$
\left.\frac{d}{d y} \Phi(t, y)\right]_{y=y_{0}}-q_{i} \Phi\left(t, y_{0}\right)=0
$$

The latter two equations determine both the eigenvalues $t_{n}$ and the coefficient $A\left(t_{n}\right)$. Using $(22)$ and $(25)$, it is seen that

$$
A(t)=-\left[\frac{w_{1}^{\prime}\left(t-y_{0}\right)+q_{i} w_{1}\left(t-y_{0}\right)}{w_{2}^{\prime}\left(t-y_{0}\right)+q_{i} w_{2}\left(t-y_{0}\right)}\right]
$$

where the prime indicates a derivative with respect to the argument $t-y_{0}$ of the Airy function. If (24) is also to be satisfied, it follows that

where

$$
A\left(t_{n}\right) B\left(t_{n}\right)=1=e^{-i 2 \pi n},
$$

$$
B(t)=-\left[\frac{w_{2}^{\prime}(t)-q w_{2}(t)}{w_{1}^{\prime}(t)-q w_{1}(t)}\right]
$$

Within the approximations used, the modal equation (27) for this cylindrical model is identical to the one developed for a concentric spherical model [Wait, 1961].

It should be mentioned at this stage that the surface $r=a+h$ can be regarded as a reference surface where the ratio of the tangential fields is specified. In the general case, $Z_{i}$ or $q_{i}$ may be a function of the eigenvalues $t_{n}$. However, for VLF it is a good approximation to regard $Z_{i}$ or $q_{i}$ as constants. This is equivalent to stating that the surface impedance does not depend on the angle of incidence. In a similar manner $Z_{g}$ or $q$ can be regarded as constants.

The orthogonality properties of the modes are now studied. We consider two sets of values, $t_{n}$ and $t_{m}$, which satisfy the boundary eqs (14a) and (14b). However, for any value of $y$ these must also satisfy

$$
\frac{d^{2}}{d y^{2}} \Phi_{n}-\left(t_{n}-y\right) \Phi_{n}=0, \Phi_{n}=\Phi\left(t_{n}, y\right),
$$

and

$$
\frac{d^{2}}{d y^{2}} \Phi_{m}-\left(t_{m}-y\right) \Phi_{m}=0, \Phi_{m}=\Phi\left(t_{m}, y\right)
$$

After multiplying the first of these equations by $\Phi_{m}$ and the second by $\Phi_{n}$, they are subtracted from one another. Both sides of the resulting equations are then integrated with respect to $y$ over the range 0 to $y_{0}$. This results in

$$
\left.\Phi_{n} \frac{d}{d y} \Phi_{m}-\Phi_{m} \frac{d}{d y} \Phi_{n}\right]_{0}^{y_{0}}=\left(t_{n}-t_{m}\right) \int_{0}^{y_{0}} \Phi_{n} \Phi_{m} d y
$$

In view of the boundary conditions on $\Phi_{n}$ and $\Phi_{m}$ at $y=0$ and $y=y_{0}$, the left-hand side of the preceding equation is zero. Thus, the integral on the right also vanishes if $t_{n}$ is not equal to $t_{m}$. Therefore, we have the important result

$$
\int_{0}^{y_{0}} \Phi\left(t_{m}, y\right) \Phi\left(t_{n}, y\right) d y=0 \text { if } m \neq n \text {. }
$$

It now follows that, if both sides of (21) are multiplied by $\Phi\left(t_{m}, y_{0}\right)$ and integrated from 0 to $y_{0}$,

The normalizing integral

$$
b_{n}=\frac{\int_{0}^{y_{0}} H\left(x_{0}, y\right) \Phi\left(t_{n}, y\right) d y}{\int_{0}^{y_{0}}\left[\Phi\left(t_{n}, y\right)\right]^{2} d y}
$$

$$
N_{n}=\int_{0}^{y_{0}}\left[\Phi\left(t_{n}, y\right)\right]^{2} d y,
$$


is now expressed in a more convenient form. This is accomplished by noting that

$$
\int\left[\Phi\left(t_{n}, y\right)\right]^{2} d y=-\left(t_{n}-y\right)\left[\Phi\left(t_{n}, y\right)\right]^{2}+\left[\Phi^{\prime}\left(t_{n}, y\right)\right]^{2} .
$$

This can be proved by differentiating both sides with respect to $y$ and making use of (29). Then using the definition of $\Phi\left(t_{n}, y\right)$ in terms of Airy functions, it follows that

and

$$
\Phi\left(t_{n}, 0\right)=-\frac{2 i}{w_{2}^{\prime}\left(t_{n}\right)-q w_{2}\left(t_{n}\right)},
$$

$$
\Phi\left(t_{n}, y_{0}\right)=-\frac{2 i}{w_{2}^{\prime}\left(t_{n}-y_{0}\right)+q_{i} w_{2}\left(t_{n}-y_{0}\right)},
$$

where use is also made of the Wronskian condition

$$
w_{1}(t) w_{2}^{\prime}(t)-w_{1}^{\prime}(t) w_{2}(t)=-2 i
$$

which is valid for any value of $t$. Finally, on making use of (35), (36), and (37) along with (14a) and $(14 \mathrm{~b})$, it is found that

$$
N_{n}=-\frac{4\left(t_{n}-q^{2}\right)}{\left[w_{2}^{\prime}\left(t_{n}\right)-g w_{2}\left(t_{n}\right)\right]^{2}}+\frac{4\left(t_{n}-y_{0}-q_{i}^{2}\right)}{\left[w_{2}^{\prime}\left(t_{n}-y_{0}\right)+q_{i} w_{2}\left(t_{n}-y_{0}\right)\right]^{2}} .
$$

This can be regarded as a fairly important result.

Equation (33) for the coefficient $b_{n}$ can be written in the convenient form

$$
b_{n}=\frac{2 \Lambda_{n}}{y_{0}} \frac{\int_{0}^{y_{0}} H\left(x_{0}, y\right) \Phi\left(t_{n}, y\right) d y}{\left[\Phi\left(t_{n}, 0\right)\right]^{2}},
$$

where

$$
\Lambda_{n}=\frac{y_{0}}{2}\left[\left(t_{n}-q^{2}\right)-\left(t_{n}-y_{0}-q_{i}^{2}\right)\left(\frac{w_{2}^{\prime}\left(t_{n}\right)-q w_{2}\left(t_{n}\right)}{w_{2}^{\prime}\left(t_{n}-y_{0}\right)+q_{i} w_{2}\left(t_{n}-y_{0}\right)}\right)^{2}\right]^{-1} .
$$

We are now in the position to obtain the fields resulting from a line dipole source at height $\hat{z}$ above the earth's surface. Thus, the aperture field is specified by

$$
H(r, 0)=\delta(z-\hat{z})(I / 2) d l,
$$

where $z=r-a$ and where $I$ and $d l$ have the same meaning as used previously. In terms of the natural coordinates, the assumed field in the aperture plane is given by

$$
H\left(x_{0}, 0\right)=k\left(\frac{2}{k a}\right)^{1 / 3} \delta(y-\hat{y}) \frac{I}{2} d l,
$$

where $\hat{y}=\left(\frac{2}{k a}\right)^{1 / 3} k \hat{z}$. Using this result and (40), it follows that

$$
b_{n}=I \frac{\Lambda_{n}}{y_{0}} \frac{\Phi\left(t_{n}, \hat{y}\right)}{\left[\Phi\left(t_{n}, 0\right)\right]^{2}}\left(\frac{2}{k a}\right)^{1 / 3} k
$$

The resultant field for a dipole line source, at $\theta_{0}=0$, may now be written compactly in the form

$$
H(x, y)=H^{(0)}(x, y) V
$$

where

$$
V=\frac{4(\pi x)^{1 / 2}}{y_{0}} e^{-i \pi / 4} \sum_{n=1,2,3} e^{-i x t}{ }_{n} \Lambda_{n} G_{n}(\hat{y}) G_{n}(y),
$$




$$
\begin{gathered}
H^{(0)}(x, y)=\frac{I d l(i k)^{1 / 2}}{2(2 \pi a \theta)^{1 / 2}} e^{-i k a \theta}=\frac{I d l e^{i \pi / 4}}{4(\pi x)^{1 / 2}}\left(\frac{2}{k a}\right)^{1 / 3} k e^{-i k a \theta}, \\
G_{n}(y)=\frac{\Phi\left(t_{n}, y\right)}{\Phi\left(t_{n}, 0\right)}
\end{gathered}
$$

and

$$
G_{n}(\hat{y})=\frac{\Phi\left(t_{n}, \hat{y}\right)}{\Phi\left(t_{n}, 0\right)}
$$

The quantity $H^{(0)}$, defined above, is numerically equal to the broad-side field of the line dipole source in free space at a distance equal to $a \theta$ (e.g., compare with (4) for $\alpha=\pi / 2$ ). The quantity $G_{n}(y)$, described as a height-gain function, is the ratio of the field of mode $n$ at height $y$ to the field of the same mode at $y=0$.

The coefficient $\Lambda_{n}$, which is defined by (41), is called the "excitation factor." The physical significance of this factor may be evident from the identity

$$
\Lambda_{n}=\frac{y_{0}}{2 \int_{0}^{y_{0}}\left[G_{n}(y)\right]^{2} d y},
$$

which may be verified by comparing the right-hand sides of (33) and (40). As may be seen, height-gain functions $G_{n}(y)$, which increase appreciably with height, correspond to small excitation factors.

The expression for $V$ given above is identical in form to the corresponding result derived for a spherical model under the assumption that $h / a<<1$ and $k a>>1$. In both cases, $V$ may be described as the ratio of the actual field to a "primary field." Furthermore, the heightgain function $G_{n}(y)$ and the excitation factor $\Lambda_{n}$ also have identical definitions in the cylindrical and spherical models.

\section{Extension to Waveguides of Nonuniform Width}

The development of the theory in this paper lends itself quite readily to certain generalizations. A case of some interest is when the height of the upper boundary varies in the direction of propagation. In the two-dimensional model considered here, the (normalized) height $y_{0}\left(x^{\prime}\right)$ is considered to be a function of $x^{\prime}$, the (normalized) distance. As indicated in figure 3 , $y_{0}\left(x^{\prime}\right)$ varies, in a smooth manner, from $y_{0}(0)$ above the source to $y_{0}(x)$ above the observer. The (normalized) surface impedances of the walls are also regarded as a slowly varying function of $x^{\prime}$.

On physical grounds it can be expected that a waveguide with slowly varying properties will not differ fundamentally from a waveguide of constant cross section. In other words, at a distance $x^{\prime}$, the structure of the modes is characteristic of a uniform waveguide of constant

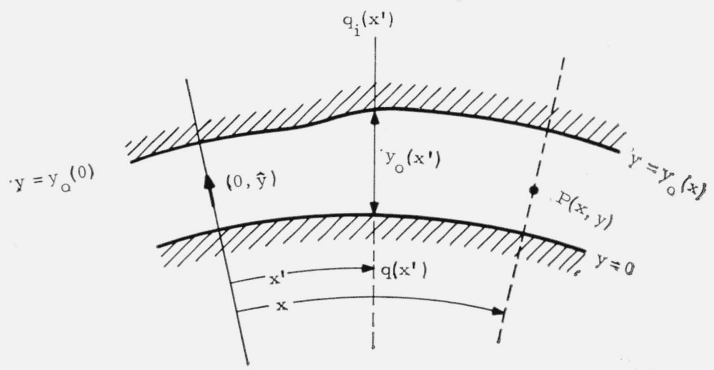

Figure 3. Waveguide of nonuniform width. 
width which is equal to $y_{0}\left(x^{\prime}\right)$ and with constant wall impedances equal to $q\left(x^{\prime}\right)$ and $q_{i}\left(x^{\prime}\right)$. To obtain the overall behavior of the field one is led to integrate over the whole range of $x^{\prime}$ from 0 to $x$. Thus, the appropriate form of the (complex) phase term is

$$
\exp \left[-i \int_{0}^{x} t_{n}\left(x^{\prime}\right) d x^{\prime}\right] \exp (-i k a \theta)
$$

where $x=(k a / 2)^{1 / 3} \theta, x^{\prime}=(k a / 2)^{1 / 3} \theta^{\prime}$, and $t_{n}\left(x^{\prime}\right)$ is the slowly varying eigenvalue. From what we have said above, it follows that $t_{n}\left(x^{\prime}\right)$ is a solution of

$$
A\left[t_{n}\left(x^{\prime}\right)\right] B\left[t_{n}\left(x^{\prime}\right)\right]=\exp (-i 2 \pi n),
$$

where $A\left[t_{n}\right]$ and $B\left[t_{n}\right]$ have the same definitions as (26) and (28), where now $y_{0}, q$, and $q_{i}$ are functions of $x^{\prime}$.

For the slowly varying waveguide, it is also necessary to employ the height-gain functions determined by the local width. Thus, $G_{n}(y)$ is to be replaced by the function

$$
G_{n}\left(x^{\prime}, y\right)=\frac{\Phi\left[t_{n}\left(x^{\prime}\right), y\right]}{\Phi\left[t_{n}\left(x^{\prime}\right), 0\right]}
$$

which is an obvious generalization of (48).

The resultant expression for the field is thus given by

where

$$
H(x, y)=H^{(0)}(x, y) \tilde{V}
$$

$$
\tilde{V}=\frac{4(\pi x)^{1 / 2}}{\left[y_{0}(0) y_{0}(x)\right]^{1 / 2}} e^{-i \pi / 4} \sum_{n=1,2,3, \ldots} \exp \left[-i \int_{0}^{x} t_{n}\left(x^{\prime}\right) d x^{\prime}\right] \tilde{\Lambda}_{n} G_{n}(0, \hat{y}) G_{n}(x, y) .
$$

The "excitation factor" is now obtained from

$$
\tilde{\Lambda}_{n}=\frac{\left[y_{0}(0) y_{0}(x)\right]^{1 / 2}}{2\left\{\int_{0}^{y_{0}(0)}\left[G_{n}(0, y)\right]^{2} d y \int_{0}^{y_{0}(x)}\left[G_{n}(x, y)\right]^{2} d y\right\}^{1 / 2}},
$$

which is an obvious generalization of (50). It is then evident that

$$
\tilde{\Lambda}_{n}=\left[\Lambda_{n}^{(0)} \Lambda_{n}^{(x)}\right]^{1 / 2},
$$

where $\Lambda_{n}^{(0)}$ is defined by (41) if $y_{0}, q$, and $q_{i}$ are replaced by $y_{0}(0), q(0)$, and $q_{i}(0)$, respectively. Similarly, the definition of $\Lambda_{n}^{(x)}$ has the form of (41) if the corresponding quantities $y_{0}(x)$, $q(x)$, and $q_{i}(x)$ are employed.

It might be mentioned that if the curvature of the walls is sufficiently small and $q_{i}$ is essentially zero, $\tilde{\Lambda}_{n}$ will become equal to unity as in the guide of constant width [Wait, 1962b]. Thus, in this limiting case, it may be seen from (54) that the fields of the individual modes vary approximately as $1 / \sqrt{y_{0}(x)}$, apart from the exponential factors. This behavior is consistent with conservation of power since the outward power flow is proportional to $1 / y_{0}(x)$ or inversely to the width of the waveguide.

The form of (54) is suggestive of a WKB approximation for propagation in a horizontally stratified medium with slowly varying properties. Implicit in the development is the assumption that conversion of modes from one order to another may be ignored. Thus, the properties of the waveguides must vary only slightly in a distance equal to one wavelength.

\section{Waveguide With Localized Obstruction}

If the width of the waveguide changes suddenly, it is apparent that the results in the previous section are not valid. In this case, it is suggested that a Kirchhoff-Huygen's ap- 


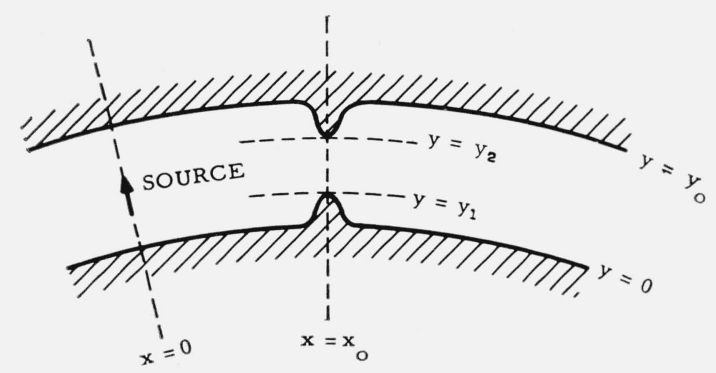

FIGURE 4. The concentric cylindrical waveguide with a localized obstruction in the aperture plane $\mathrm{x}=\mathrm{x}_{0}$.

proach is useful [Wait, 1962c].

It is now imagined that the incident field results from an equivalent line magnetic source at $x=0$ (i.e., $\theta=0$ ). Thus,

$$
H(x, y)=\sum_{m} a_{m} \Phi\left(t_{m}, y\right) e_{m}^{-i x t} \text { for } x<x_{0}
$$

where $a_{m}$ is a coefficient which does not depend on $x$ or $y$. It is assumed that the aperture plane $x=x_{0}$ is obstructed in such a manner that the effective aperture is a slit extending from $y=y_{1}$ to $y_{2}$ (i.e., $r-a=z_{1}$ to $z_{2}$ ). The situation is illustrated in figure 4 . Thus, within the Kirchhoff approximation,

$$
\begin{aligned}
H\left(x_{0}, y\right) & =\sum a_{m} \Phi\left(t_{m}, y\right) e^{-i x_{0} t_{m}} \text { for } y_{1}<y<y_{2} \\
& =0 \text { for } 0<y<y_{1} \\
& =0 \text { for } y_{2}<y<y_{0} .
\end{aligned}
$$

In other words, we are assuming that the field within the aperture of the slit has the same value as if the slit were not present. It is known from a study of the rigorous solutions of diffraction by slits that this is an excellent approximation [Born and Wolf, 1959] provided the width of the slit is greater than about a wavelength.

The field in the region $x>x_{0}$ can now be expressed in the form

$$
H(x, y)=\sum_{m} \sum_{n} A_{n}^{(m)} \Phi\left(t_{n}, y\right) e^{-i\left(x-x_{0}\right) t} n e^{-i x_{0} t_{m}}
$$

where

$$
A_{n}^{(m)}=\frac{2 \Lambda_{n}}{y_{0}} \frac{\int_{y_{1}}^{y_{2}} \Phi\left(t_{m}, y\right) \Phi\left(t_{n}, y\right) d y}{\left[\Phi\left(t_{n}, 0\right)\right]^{2}} a_{m} .
$$

We see clearly that the incident mode of order $m$ excites modes of order $n$ where $m$ and $n$ are positive integers.

It is convenient to write

where, for $m \neq n$,

$$
A_{n}^{(m)}=\left[P_{n}^{(m)}+Q_{n}^{(m)}\right] a_{m}
$$

$$
P_{n}^{(m)}=-\frac{2 \Lambda_{n}}{y_{0}} \frac{\Phi\left(t_{m}, 0\right)}{\Phi\left(t_{n}, 0\right)} \int_{0}^{y_{1}} G_{m}(y) G_{n}(y) d y
$$

and

$$
Q_{n}^{(m)}=-\frac{2 \Lambda_{n}}{y_{0}} \frac{\Phi\left(t_{m}, 0\right)}{\Phi\left(t_{n}, 0\right)} \int_{y_{2}}^{y_{0}} G_{m}(y) G_{n}(y) d y
$$

where

$$
G_{n}(y)=\frac{\Phi\left(t_{n}, y\right)}{\Phi\left(t_{n}, 0\right)}
$$


and

$$
G_{m}(y)=\frac{\Phi\left(t_{m}, y\right)}{\Phi\left(t_{m}, 0\right)}
$$

In obtaining the above forms for $P_{n}^{(m)}$ and $Q_{n}^{(m)}$ use has been made of the orthogonality condition given by (32).

When $m=n$, we have

where

$$
A_{n}^{(n)}=\left[P_{n}^{(n)}+Q_{n}^{(n)}\right] a_{n}
$$

$$
P_{n}^{(n)}=1-\frac{2 \Lambda_{n}}{y_{0}} \int_{0}^{y_{1}}\left[G_{n}(y)\right]^{2} d y
$$

and

$$
Q_{n}^{(n)}=1-\frac{2 \Lambda_{n}}{y_{0}} \int_{y_{2}}^{y_{0}}\left[G_{n}(y)\right]^{2} d y
$$

where use has been made of (39).

The integrals over the range 0 to $y_{1}$ in the preceding equations can be regarded as the influence of the obstacle on the ground, whereas the integrals over $y_{2}$ to $y_{0}$ are related to the protuberance at the ionosphere. To evaluate these integrals it is desirable to expand the $G$ functions as a power series in $y$.

Since

$$
\begin{gathered}
G_{n}(0)=1 \\
{\left[d G_{n}(y) / d y\right]_{y=0}=-q}
\end{gathered}
$$

and

$$
\frac{d^{2} G(y)}{d y^{2}}=\left(t_{n}-y\right) G(y) \text { for any } y,
$$

it is not difficult to show that

$$
G_{n}(y)=1-q y+\frac{t_{n} y^{2}}{2}-\frac{\left(1+t_{n} q\right)}{6} y^{3}+\ldots
$$

Thus,

$$
G_{n}(y) G_{m}(y)=1-2 q y+\left(t_{n}+t_{m}+2 q^{2}\right) \frac{y^{2}}{2}-\left(1+2 t_{n} q+2 t_{m} q\right) \frac{y^{3}}{3}+\ldots
$$

and the expansion for $\left[G_{n}(y)\right]^{2}$ is obtained by simply replacing $t_{m}$ by $t_{n}$ in the preceding result. The integrations for the $P$ integrals are now readily carried out. They yield

where

$$
P_{n}^{(m)}=-\frac{2 \Lambda_{n}}{y_{0}} g_{m, n}\left[y_{1}-q y_{1}^{2}+\left(t_{n}+t_{m}+2 q^{2}\right) \frac{y_{1}^{3}}{6}-\left(1+2 t_{n} q+2 t_{m} q\right) \frac{y_{1}^{4}}{12}+\ldots\right]
$$

$$
g_{m, n}=\frac{\Phi\left(t_{m}, 0\right)}{\Phi\left(t_{n}, 0\right)}=\frac{w_{2}^{\prime}\left(t_{n}\right)-q w_{2}\left(t_{n}\right)}{w_{2}^{\prime}\left(t_{m}\right)-q w_{2}\left(t_{m}\right)}
$$

and

$$
P_{n}^{(n)}=1-\frac{2 \Lambda_{n}}{y_{0}}\left[y_{1}-q y_{1}^{2}+\left(t_{n}+q^{2}\right) \frac{y_{1}^{3}}{3}-\left(1+4 t_{n} q\right) \frac{y_{1}^{4}}{12}+\ldots\right] \text {. }
$$

The $Q$ integrals are evaluated in a very similar manner. Thus

$$
\begin{aligned}
Q_{n}^{(m)}=-\frac{2 \Lambda_{n}}{y_{0}} g_{m, n} G_{m}\left(y_{0}\right) G_{n}\left(y_{0}\right)[ & \left(y_{0}-y_{2}\right)-q_{i}\left(y_{0}-y_{2}\right)^{2} \\
& \left.+\left(t_{n}+t_{m}+2 q_{i}^{2}\right) \frac{\left(y_{0}-y_{2}\right)^{3}}{6}-\left(1+2 t_{n} q_{i}+2 t_{m} q_{i}\right) \frac{\left(y_{0}-y_{2}\right)^{4}}{12}+\ldots\right]
\end{aligned}
$$

and

$$
Q_{n}^{(n)}=1-\frac{2 \Lambda_{n}}{y_{0}}\left[G_{n}\left(y_{0}\right)\right]^{2}\left[\left(y_{0}-y_{2}\right)-q_{i}\left(y_{0}-y_{2}\right)^{2}+\left(t_{n}+q_{i}^{2}\right) \frac{\left(y_{0}-y_{2}\right)^{3}}{3}-\left(1+4 t_{n} q_{i}\right) \frac{\left(y_{0}-y_{2}\right)^{4}}{12}+\ldots\right] .
$$


Due to the typically large values of $q_{i}$ at VLF, the preceding series for the $Q$ functions are probably not useful. It would be better to work directly with eqs (62) and (67).

\section{Discussion of Formulas}

Some of the previous results are now discussed briefly. For purposes of illustration, it is assumed that the ionosphere is a sharply bounded medium whose effective conductivity is $\epsilon_{0} \omega_{r}$ where $\epsilon_{0}=8.854 \times 10^{-12}$. Under this condition extensive numerical values of the coefficients $t_{n}$ satisfying eq (27) are available [Spies and Wait, 1961]. Using these values, the various quantities entering into the formulas for the modal coefficients can be evaluated in a straightforward manner.

It is seen that the modal coefficients, given by eqs (73), (75), (76), and (77) all contain the factor $\Lambda_{n}$. This factor is a modal excitation factor and it is a measure of the efficiency of excitation of a given mode from a line or dipole source [Wait, 1961, 1962b]. In the present context it is normalized so that it approaches unity for perfect ground conductivity $(g=0)$ and a flat earth $(a=\infty)$. In general it is a complex quantity. To illustrate its behavior $\omega_{r}$ is set equal to $2 \times 10^{5}$ and $h$ is taken as $70 \mathrm{~km}$. Furthermore, the ground is assumed to be perfectly conducting. Under these conditions $\Lambda_{n}$ for $n=1$ has the following complex values for the frequencies indicated:

$$
\begin{array}{rlrl}
\Lambda_{1}= & 0.95 \underline{\mid 3.8^{\circ}}(10 \mathrm{kc} / \mathrm{s}), & 0.79 \underline{\mid 3.0^{\circ}}(15 \mathrm{kc} / \mathrm{s}), \\
& 0.59 \underline{4.6^{\circ}}(20 \mathrm{kc} / \mathrm{s}), & 0.37 \underline{7.4^{\circ}}(25 \mathrm{kc} / \mathrm{s}), \\
& 0.19 \underline{10.6^{\circ}(30 \mathrm{kc} / \mathrm{s}) .} & &
\end{array}
$$

This mode corresponds to the mode of least attenuation. It is characterized by an excitation factor which decreases approximately as the inverse of the frequency. Under the same conditions $\Lambda_{n}$, for $n$ greater than 1, is roughly unity over the same frequency range [Wait, $1962 \mathrm{~b}]$.

The modal coefficient $P_{n}^{(n)}$ defined by (75), in the case of $n=1$, can be written

$$
P_{1}^{(1)} \cong 1-2 \Lambda_{1} \frac{h_{1}}{h}
$$

Here, $h_{1} / h$ is the ratio of the heights of the obstacle on the ground to the height of the ionosphere. This quantity would never be greater than about 0.05 and thus the modification of the first mode by even an extremely high mountain range would be small. This is particularly the case at the upper end of the VLF band where the excitation factor is small.

The relative conversion of the field from an incident mode of order 1 to a mode of order 2 is obtained from the factor $P_{n}^{(m)}$ defined by (73) for $m=1$ and $n=2$. Approximately, this can be written

$$
P_{2}^{(1)} \cong-2 \Lambda_{2} g_{1,2}\left(h_{1} / h\right) \text {. }
$$

The complex quantity $g_{1,2}$ is defined by eq (74) for $m=1, n=2$, and $q=0$. For the same conditions, its magnitude for the frequencies indicated, are given as follows

$$
\left|g_{1,2}\right|=1.72(10 \mathrm{kc} / \mathrm{s}), 1.47(15 \mathrm{kc} / \mathrm{s}), 1.08(20 \mathrm{kc} / \mathrm{s}), 0.70(25 \mathrm{kc} / \mathrm{s}) \text {, and } 0.41(30 \mathrm{kc} / \mathrm{s}) .
$$

Since $\left|\Lambda_{2}\right|$ is of the order of unity, it is thus apparent that the conversion to higher modes may be significant.

The influence of the protuberance on the upper boundary is described by eqs (76) and (77). The situation is similar to that of the ground obstacle except that the factors $G_{n}\left(y_{0}\right)$ appear. Actually, these are the ratio of the field just below the ionosphere reflecting layer to the field at the ground.

Of particular interest is the possibility that, as a consequence of an ionospheric irregularity, a mode of order 1 may be excited by an incident mode of order 2. The magnitude of this 
first-order mode relative to amplitude of the second-order mode is obtained from eq (76) with $m=2$ and $n=1$. Thus, approximately

$$
Q_{1}^{(2)} \cong-2 \Lambda_{1} g_{2,1} G_{2}\left(y_{0}\right) G_{1}\left(y_{0}\right)\left(\frac{h-h_{2}}{h}\right) .
$$

The numerical magnitude of the excitation factor $\Lambda_{1}$ has already been discussed. As noted, it may be quite small for frequencies of the order of $25 \mathrm{kc} / \mathrm{s}$. However, in certain cases, the height-gain function $G_{1}(y)$, is somewhat greater than unity. Thus, the conversion to the lower-order mode may be quite significant. This point can also be demonstrated directly from eq (62) which, in this special case, has the form

$$
Q_{1}^{(2)}=-\frac{2 \Lambda_{1}}{y_{0}} g_{2,1} \int_{y_{2}}^{y_{0}} G_{2}(y) G_{1}(y) d y .
$$

Modes of the "whispering gallery" type [Budden and Martin, 1962], also known as "earthdetached modes" [Wait, 1962b], are associated with a low excitation efficiency (i.e., $\Lambda_{1}$ is small). However, the height-gain function $G_{1}(y)$ for a "whispering gallery mode" is an increasing function of height. Thus, the product of the integral over $y_{2}$ to $y_{0}$ and the excitation factor $\Lambda_{1}$ may be of appreciable magnitude.

Further work on this subject awaits the completion of the extensive tabulations of numerical values of the coefficients $t_{n}$ and the height-gain functions $G_{n}(y)$ for a range of the parameters. Also, the complications resulting from the earth's magnetic field are incorporated following the approach introduced in a previous paper [Wait, 1963].

I thank Prof. S. Maley and Mr. K. P. Spies for their helpful remarks, and Mrs. Eileen Brackett for her assistance in preparing the manuscript.

\section{References}

Born, Max and Emil Wolf (1959), Principles of optics (Pergamon Press).

Budden, K. G., and H. G. Martin (1962). The ionosphere as a whispering gallery, Proc. Roy. Soc. Ser. A, 265, 554-569.

Miller, J. C. P. (1946), The Airy integral, giving tables of solutions of the differential equation $y^{\prime \prime}=x y($ Cambridge University Press).

Spies, K. P., and J. R. Wait (July 17, 1961), Mode calculations for VLF propagation in the earth-ionosphere waveguide, NBS Technical Note No. 114.

Wait, J. R. (1959), Electromagnetic radiation from cylindrical structures (Pergamon Press).

Wait, J. R. (Jan.-Feb. 1961), A new approach to the mode theory of VLF propagation, J. Res. NBS 65D (Radio Prop.), No. 1, 37-46.

Wait, J. R. (July-Aug. 1962a), An analysis of VLF mode propagation for a variable ionosphere height, J. Res. NBS 66D (Radio Prop.), No. 4, 453-461.

Wait, J. R. (1962b), Electromagnetic waves in stratified media (Pergamon Press, Oxford, 1962, and Macmillan, New York, 1962).

Wait, J. R. (June 8, 1962c), Mode conversion in the earth-ionosphere waveguide, NBS Technical Note No. 151.

Wait, J. R. (1963), The mode theory of VLF radio propagation for a spherical earth and a concentric anisotropic ionosphere, Can. J. Phys. 41, 299-315 and 819.

Wait, J. R., and K. P. Spies (Mar--Apr. 1963), Height-gain for VLF radio waves, J. Res. NBS 6rD (Radio Prop.), No. 2, 183-187.

(Paper 68D1-320) 